\title{
Ergogenic effects of an epidural neuroprosthesis in one individual with spinal cord injury
}

Tom E. Nightingale, PhD, Matthias Walter, MD, PhD, Alison M.M. Williams, BKin, Tania Lam, PT, PhD, and Andrei V. Krassioukov, MD, PhD

Neurology ${ }^{\circledR}$ 2019;92:338-340. doi:10.1212/WNL.0000000000006923
Correspondence

Dr. Krassioukov

krassioukov@icord.org

Individuals with a spinal cord injury $(\mathrm{SCI}) \geq \mathrm{T} 6$ level exhibit impaired cardiovascular responses to exercise that can limit exercise capacity. ${ }^{1}$ The burgeoning field of neuromodulation has recently demonstrated that epidural spinal cord stimulation (ESCS), configured to facilitate motor function in humans with SCI, can also modulate cardiovascular function (i.e., increasing or maintaining arterial blood pressure at rest or in response to an orthostatic challenge, respectively). ${ }^{2-4}$ Here we present a unique application of ESCS to improve upper body exercise capacity in an individual with SCI, via the modulation of cardiovascular and respiratory function.

\section{Case description}

A 33-year-old man with a chronic (5 years) motor-complete SCI (American Spinal Injury Association Impairment Scale B) at C5 was fitted 18 months prior to this study with a neurostimulator and 16-electrode array (Restore-ADVANCED and Specify 5-6-5; Medtronic; medtronic.com/us-en/healthcare-professionals/products/neurological/spinal-cord-stimulation-systems/restoreadvanced-surescan-mri-neurostimulator.html) between $\mathrm{T} 11$ and $\mathrm{L} 1$ (figure 1A). We conducted testing over a 3-month period, with an initial assessment of trunk/ lower limb EMG (figure 1C). These preliminary findings allowed us to determine the optimal parameters to target (1) trunk activation (abdominal program) and (2) trunk and lower limb activation while also identifying the most appropriate amplitudes for low- and high-intensity stimulation (figure 1B). The latter ESCS program has previously been shown to modulate cardiovascular function at rest in this individual ${ }^{4}$ and will be referred to as the cardiovascular stimulation program.

The participant performed 6 progressive upper body exercise tests on an arm-crank ergometer until volitional exhaustion, each separated by $\sim 12$ days. During each session, the participant was randomly assigned to 4 different ESCSs (figure 1B) or no ESCS (control) $(n=2)$. We blinded assessors to the trial allocation and kept verbal motivation consistent between trials. A detailed description, along with photographs of the test day protocol, is available in figure e-1 (doi.org/ 10.5061/dryad.5pp17kd). Prior to exercise, both before and immediately after stimulation (ESCS) or perceived stimulation (no ESCS), we measured brachial blood pressure (BP). The primary outcome measure during exercise was peak oxygen uptake $\left(\mathrm{V}_{2}\right.$ peak) (assessed via an online metabolic cart). We also calculated peak oxygen pulse and ventilation, along with episodically assessing the participant's self-reported global rating of perceived exertion (RPE).

Relative to control trials, ESCS improved absolute and relative $\mathrm{VO}_{2}$ peak (15\%-26\%), irrespective of stimulation intensity and electrode configuration (figure $1, \mathrm{D}$ and $\mathrm{E}$ ). Peak oxygen pulse increased with ESCS (figure $1 \mathrm{~F}$ ), implying improved stroke volume (SV). While the abdominal ESCS program effected peak minute ventilation (figure $1 G$ ), the cardiovascular program altered mean arterial pressure (MAP) (figure $1 \mathrm{H})$. The participant's global RPE was also lower for a given power output $(60 \mathrm{~W})$ with ESCS compared to control (figure 1I). 
Figure 1 Epidural spinal cord stimulation (ESCS) configuration and cardiorespiratory outcomes during exercise
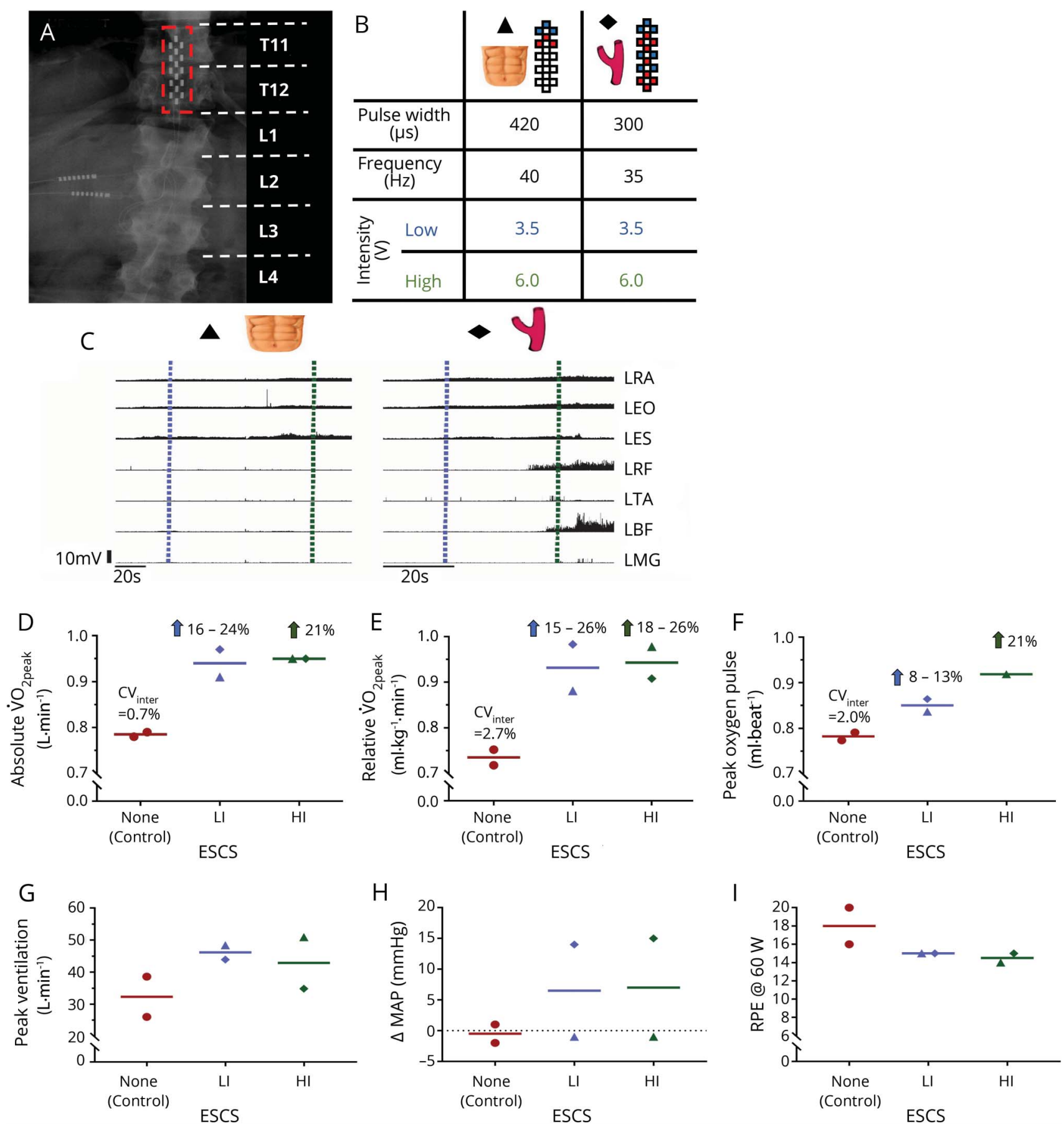

(A) Conventional X-ray confirms anatomical position (vertebrae levels T11-L1) of 16-electrode array. (B) Preset stimulation parameters and electrode configurations (red is cathode, blue is anode, white is inactive) target specific muscle groups as demonstrated in panel (C). $\mathbf{\Delta}$ Denotes abdominal-specific program. Denotes cardiovascular-specific program. Preliminary surface EMG testing was performed in a seated position, whereby a concurrent stepwise increase in stimulation intensity (i.e., 1, 1.5, 2, 2.5 V) was applied for each electrode configuration (C). Blue dashed line indicates low-intensity (LI; beneath the threshold to elicit first motor activation) stimulation. Green dashed line indicates high-intensity (HI; highest intensity tolerated) stimulation. Absolute (D) and relative (E) $\mathrm{VO}_{2}$ peak and peak oxygen pulse (F) values were similar during no ESCS trials (as demonstrated by the negligible intertrial coefficients of variation [CV $\left.{ }_{\text {inter }}\right]$ ), yet were improved with ESCS. Percentage change values with ESCS relative to control trials are displayed. It was not possible to calculate peak oxygen pulse for one HI ESCS trial due to heart rate monitor malfunction. (G) Robust differences, relative to control trials, were observed in peak ventilation using the abdominal stimulation program. (H) The cardiovascular ESCS program increased mean arterial pressure (MAP) by $14 \mathrm{~mm} \mathrm{Hg}$ prior to exercise, with no additive effect of increasing stimulation intensity. (I) RPE for a given power output (60 W) was also lower with ESCS. LBF = left bicep femoris; LEO = left external obliques; LES = left erector spinae; LMG = left medial gastrocnemius; LRA = left rectus abdominis; LRF = left rectus femoris; LTA = left tibialis anterior; RPE = rating of perceived exertion. 


\section{Discussion}

We demonstrate that acute ESCS effectively and safely improves exercise capacity during upper body exercise in an individual with motor-complete tetraplegia. As a result of decreased vascular and skeletal muscle tone, individuals with such injuries experience increased blood pooling and decreased venous return to the heart, which has a detrimental effect on SV. This consequently blunts cardiac output, the major limiting factor for $\dot{\mathrm{VO}}_{2}$ peak, and results in premature fatigue. Oxygen pulse, which noticeably increased with ESCS, depends on the size of SV and arteriovenous oxygen difference. Given oxygen extraction is maximal and relatively constant at peak, oxygen pulse therefore becomes a reasonable surrogate for SV. Previously it has been shown that ESCS can resolve orthostatic hypotension (also preserving SV and cardiac output). ${ }^{4}$ However, this is the first study to demonstrate that the instantaneous regulation of cardiovascular function via ESCS can positively affect upper body exercise performance.

Different stimulation configurations act through seemingly distinct mechanisms. The abdominal program improved the rate and depth of respiration, potentially promoting venous return via the respiratory pump. In contrast, the cardiovascular program increased MAP prior to exercise, thereby improving perfusion of skeletal muscle, which may increase metabolic activity and consequently venous return. Irrespective of the proposed underlying mechanisms, these seminal findings demonstrate that improvements in exercise capacity, commonly seen with weeks or months of aerobic exercise training, can be achieved acutely with ESCS.

Interestingly, these effects were independent of stimulation intensity, and thus skeletal muscle activation, suggesting ESCS potentially restores supraspinal respiratory and vasomotor control via increasing the resting membrane potential of spinal circuits. ${ }^{5}$ Transcutaneous spinal cord stimulation (TSCS) has also recently demonstrated augmented $\mathrm{BP}$ responses in persons with $\mathrm{SCI}^{6}$ and activates the same common neural input structures as ESCS. ${ }^{7}$ Therefore, TSCS offers an exciting possibility to noninvasively access spinal circuitry and future research should assess whether this neuromodulation modality has similar ergogenic effects as those noted in the current study for ESCS.

\section{Author contributions}

T.E. Nightingale: lead author, study and concept design, data acquisition, analysis and interpretation, writing first draft, critical revision of the manuscript. M. Walter: study and concept design, data acquisition and interpretation, critical revision of the manuscript. A.M.M. Williams: EMG data acquisition, analysis and interpretation, critical revision of the manuscript. T. Lam: EMG data acquisition, analysis and interpretation, critical revision of the manuscript. A.V. Krassioukov: study and concept design, data acquisition and interpretation, critical revision of the manuscript, study principal investigator.

\section{Study funding}

This study was funded by the Rick Hansen Institute (grant number 2015-31; Principal Investigator Dr. Krassioukov). Dr. Krassioukov's laboratory is also supported by funds from the Canadian Institutes of Health Research (CIHR), Heart and Stroke Foundation, Craig H. Neilsen Foundation, and Canadian Foundation for Innovation. Dr. Nightingale and Dr. Walter are recipients of Michael Smith Foundation for Health Research Trainee Awards in conjunction with the International Collaboration on Repair Discoveries and Rick Hansen Foundation, respectively. Alison Williams was the recipient of the 2017 Canada Graduate Scholarships-Master's Award from the CIHR.

\section{Disclosure}

The authors report no disclosures relevant to the manuscript. Go to Neurology.org/N for full disclosures.

\section{Publication history}

Received by Neurology June 30, 2018. Accepted in final form October 26, 2018.

\section{References}

1. Walter M, Krassioukov AV. Autonomic nervous system in paralympic athletes with spinal cord injury. Phys Med Rehabil Clin N Am 2018;29:245-266.

2. Aslan SC, Legg Ditterline BE, Park MC, et al. Epidural spinal cord stimulation of lumbosacral networks modulates arterial blood pressure in individuals with spinal cord injury-induced cardiovascular deficits. Front Physiol 2018;9:565.

3. Harkema SJ, Wang S, Angeli CA, et al. Normalization of blood pressure with spinal cord epidural stimulation after severe spinal cord injury. Front Hum Neurosci 2018; 12:83

4. West CR, Phillips AA, Squair JW, et al. Association of epidural stimulation with cardiovascular function in an individual with spinal cord injury. JAMA Neurol 2018; 75:630-632.

5. Capogrosso $\mathrm{M}$, Wenger N, Raspopovic S, et al. A computational model for epidural electrical stimulation of spinal sensorimotor circuits. J Neurosci 2013; 33:19326-19340.

6. Phillips AA, Squair JW, Sayenko DG, Edgerton VR, Gerasimenko Y, Krassioukov AV. An autonomic neuroprosthesis: noninvasive electrical spinal cord stimulation restores autonomic cardiovascular function in individuals with spinal cord injury. J Neurotrauma 2018;35:446-451.

7. Hofstoetter US, Freundl B, Binder H, Minassian K. Common neural structures activated by epidural and transcutaneous lumbar spinal cord stimulation: elicitation of posterior root-muscle reflexes. PLoS One 2018;13:e0192013. 


\section{Neurology}

\section{Ergogenic effects of an epidural neuroprosthesis in one individual with spinal cord injury}

Tom E. Nightingale, Matthias Walter, Alison M.M. Williams, et al. Neurology 2019;92;338-340 Published Online before print January 11, 2019

DOI 10.1212/WNL.0000000000006923

\section{This information is current as of January 11, 2019}

\section{Updated Information \&} Services

References

Permissions \& Licensing

Reprints including high resolution figures, can be found at: http://n.neurology.org/content/92/7/338.full

This article cites 7 articles, 1 of which you can access for free at: http://n.neurology.org/content/92/7/338.full\#ref-list-1

Information about reproducing this article in parts (figures,tables) or in its entirety can be found online at:

http://www.neurology.org/about/about_the_journal\#permissions

Information about ordering reprints can be found online:

http://n.neurology.org/subscribers/advertise

Neurology ${ }^{\circledR}$ is the official journal of the American Academy of Neurology. Published continuously since 1951, it is now a weekly with 48 issues per year. Copyright Copyright ( 2019 The Author(s). Published by Wolters Kluwer Health, Inc. on behalf of the American Academy of Neurology.. All rights reserved. Print ISSN: 0028-3878. Online ISSN: 1526-632X.

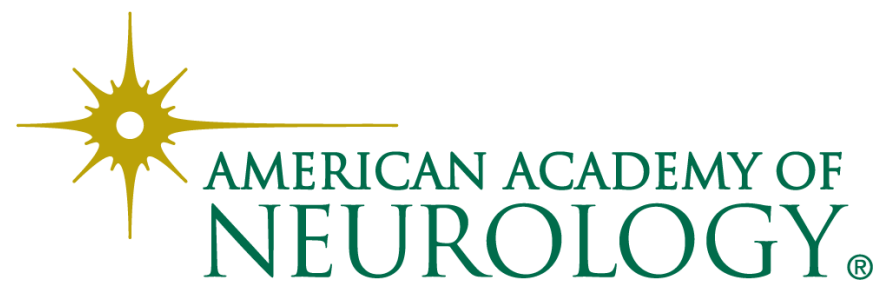

